A Course in the Geometry of $n$ Dimensions By Dr. M. G. Kendall. (Griffin's Statistical Monographs and Courses, No. 8.) Pp. viii +63 . (London: Charles Griffin and Co., Ltd., 1961.) $16 s$.

$\mathrm{W}$ ITH the rapid expansion of the application of the statistical method has come an increased demand for training in the subject by students without specialist degrees in mathematics. Such students progress more quickly if they can refer to toxt-books which are designed specifically to give those parts of mathematics required for the development of statistical theory. Dr. Kendall's book is welcome as a good and useful example of a work of this kind. By the representation of sample values from multi-variate distributions as points in $n$ dimonsional Euclidean space, as shown by Sir Ronald Fisher nearly fifty years ago, many statistical problems can be treated geometrically. This approach has ofton led to poworful and elegant results and contributes greatly to the intuitive perception of relationships.

The first two-thirds of Dr. Kendall's text presonts the basic mathematics of $n$ dimonsional geometry, dealing in particular with hypersurfaces; linear sub-spaces and the angles between these; co-ordinato transformations and the reduction of quadrics to canonical form; solid angles; the contents of hyperspheres, hyperellipsoids, hyperprisms, parallelotopes, hyperpyramids and simplices. In the final third these results are used to develop certain aspects of statistical theory, for example the chi-square, $t$ and Wishart distributions; distribution of the samplo mean from a rectangular distribution; multiple and canonical correlation and component analysis.

The descriptions and derivations are everywhere very condensed. This is not a serious drawback in the first part of the book for anyone with the reasonable knowledge of three-dimensional Euclidean geometry which is almost essential as an introduction. The contents of the second part can be studied moro thoroughly in easily available statistical texts, but it is convenient to have a unified treatment. The expansion of this part in further cditions would make the book even more valuable.

W. Brass

\section{Ordinary Differential Equations}

By L. S. Pontryagin. Translated from the Russian by Leonas Kacinskas and Walter B. Counts. (Adiwes International Series in Mathematics.) Pp. vi+298. (Reading, Mass.: Addison-Wesley Publishing Company, Inc.; London: Pergamon Press, 1962.) $55 s$.

JoW that many of the elementary devices for solving simple first-order differential equations are handled as part of a calculus course, generalizing the manipulations of integration, a genuine first course in differential equations can be made up to date and forward looking. Pontryagin's lectures, translated here, provide a strict but stimulating diot for the mathematician and for the engineer.

In the first chapter, elementary methods for first-order equations are rapidly reviewed and interest is soon concentrated on existence and uniqueness of solutions. Then the linear equation with constant coefficients is studied in detail, with elaborate illustration from olectric eircuit thoory. The concepts of phase space and trajoctories are introduced. For linear equations with periodic coefficients, Liapunov's theorem reduces a normal system to a constant coefficient system, and here the analysis demands a certain acquaintance with linear algebra and matrix theory; the essentials are given at the ond of the book, but this chapter, and its sequel on cxistence theorems, make rather heavy demands on the sophistication of the novice.

The climax of the volume is the long chapter on stability of autonomous systems (in which the independent time variable does not appear explicitly); the funda- mental Liapunov theorems are derived, and applied to the theory of Watt's centrifugal governor, the earliest of automatic control systems, and to the design of the vacuum-tube oscillator. Finally, there is a somewhat severe section on stability of periodic solutions of autonomous equations and equations with sinusoidal time terms.

Tho young mathematician should have no great difficulty in following the exposition; but the engineer may complain that the treatment is too academic. Nevertheless, if he is to master the theory of modern control engineering, with its recent spectacular exemplifications, he must master the kind of mathematics which Pontryagin presents here.

T. A. A. Broadbent

\section{Solid-Fuel Rocket Propulsion}

(Temple Pross Monographs on Rockots and Missilos.) By J. E. Daboo. Pp. vii +88 . (London: Temple Press Books, 1962.) 15s.

7 HIS little book is an excellent and well-balanced introduction to solid-propellant rockets, which, becauso they are simplo and comparatively choap, are now used widely in guided missiles and research rockets, and to a lesser extent in the launching vehicles for satellites. After reviewing the various applications and comparing solid- and liquid-fuel rockets in Chapter 1, the author expounds the thermodynamic theory of rocket gas flow in Chapter 2. Chapter 3 is dovoted to propellants-the different possible types, their burning rates, the geomotry of charge design and the problems of storage. Chapter 4 deals with the dosign of the motor case and oxit nozzle, and Chapter 5 gives a useful and realistic example of the detailed design of a typical small motor.

The book is well planned and executed; it has many good diagrams, a glossary, bibliography and index. The only fault is the rather stodgy style: the reader has to plough through a mass of abstract nouns and weak verbs, and too many phrases like "necessitate the incorporation of". But since the book is otherwise so felicitous, this defect detracts little from its value. D. G. King-Hfile

\section{Nuclear Reactions}

Vol. 2. Edited by P. M. Endt and P. B. Smith. (Series in Physies.) Pp. $x+542$. (Amsterdam: North-Holland Publishing Company, 1962.) $110 \mathrm{~s}$.

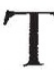
HIS volume consists of five chapters. The first, by Stewart D. Bloom, of Livermore, is on the technology of nanosecond pulsing of accelerators, and on the use of these machines for time of flight studies. The second chapter, a review of certain topies in fission physies, is by J. R. Huizenga and R. Vandenbosch, of the Argonne National Laboratory. Chapter 3, by E. G. Fuller and Evans Hayward, of the National Bureau of Standards, is a review of theoretical and experimental work on the nuclear photoeffect in the region of the giant resonance. Chapter 4 , a theoretical paper on vibrations in sphorical nucloi, is by J. M. Araujo, of tho University of Porto, while the last chapter, by Philip B. Smith, of the Rijksuniversiteit, Utrecht, consists of a vast table of angular correlation coefficients for the radiative docay of aligned nucloi. This chapter occupies 287 pages-more than half the book.

Each of these chapters is a vory excellent review or reference work, but there seems to be little or no connexion between them. It is open to argument whether the second volumo does really complete the roview of nuclear reactions started by the first; what is clear is that the second volume cannot stand on its own as a separate work sinee it lacks a simple unifying theme.

It is my opinion that for anyone who already has a copy of the first volume of Nuclear Reactions, the purchase of the second volumo would lead to a valuable increase in the scope of this useful reference book. E. R. RAE 